\title{
Atomic Mechanisms of Nanocrystallization via Cluster-Clouds in Solution Studied by Liquid-Phase Scanning Transmission Electron Microscopy
}

\author{
Walid Dachraoui,* Debora Keller, Trond R. Henninen, Olivia J. Ashton, and Rolf Erni*
}

Cite This: Nano Lett. 2021, 21, 2861-2869

Read Online

ACCESS | Lill Metrics \& More | 国 Article Recommendations ｜（s Supporting Information

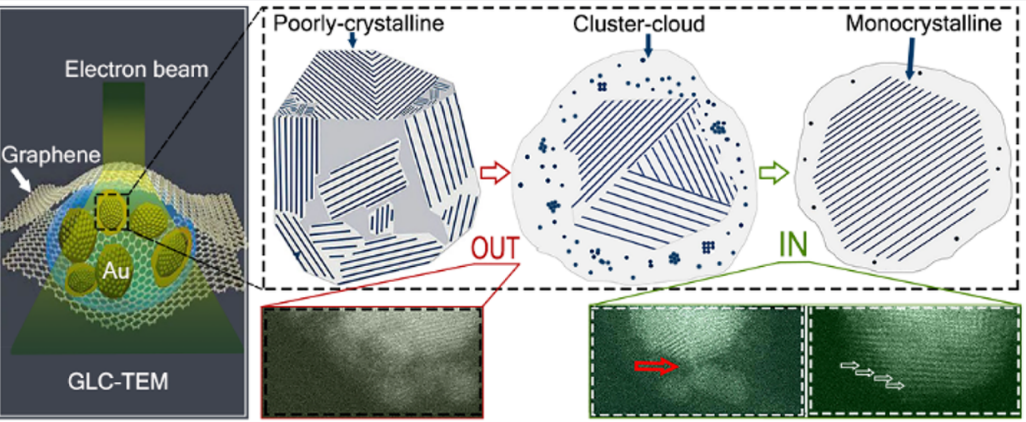

ABSTRACT: The formation of nanocrystals is at the heart of various scientific disciplines, but the atomic mechanisms underlying the early stages of crystallization from supersaturated solutions are still rather unclear. Here, we used in situ liquid-phase scanning transmission electron microscopy to study at the atomic level the very early stages of gold nanocrystal growth, and the evolution of its crystallinity. We found that the nucleation is initiated by the formation of poorly crystalline nanoparticles. These are transformed into monocrystals via nanocrystallization governed by a complex process of multiple out-and-in exchanges of matter between a crystalline-core and a disordered-shell, referred to as the cluster-cloud. Our observations at the crystal/cluster-cloud interface during growth demonstrate that the initially formed nanocrystals expel the poorly crystallized phases as nanoclusters into the cluster-cloud, then readsorb it by two distinct pathways, namely, by (i) monomer attachments and (ii) nanocluster coalescence. This growth process eventually leads to the formation of monocrystalline nanoparticles.

KEYWORDS: Gold nanoparticles, growth, in situ STEM, cluster-cloud, nanocrystallization

$\mathrm{N}$

anomaterials have received considerable attention due to their diverse applications in medicine, agriculture, energy technologies, and so forth. ${ }^{1-4}$ Understanding the formation of nanoparticles is essential for controlling their synthesis and properties. $^{5-7}$ Accordingly, there have been numerous studies on the nucleation and growth of nanoparticles, which have enabled rapid progress in nanoparticle synthesis for specific applications. $^{8}$ Despite these efforts, the early stages of crystallization are still illusive and many questions remain open. For example, the exact pathway from a molecular structure to a crystal is often missing, occurring at the atomic scale on short time scales. ${ }^{9-13}$ In the 1990s, a transmission electron microscopy (TEM) study revealed that many nanoparticles (NPs) of a few nanometers in size are in a "quasi-molten" state, in which their structure and shape fluctuate from one state to another. ${ }^{14}$ In 2005, it was reported that the growth of CdSe NPs is accompanied by crystallization and shape reconstruction of initially amorphous NPs. ${ }^{15}$ Different theoretical studies of $\mathrm{ZnS}(3.4 \mathrm{~nm})$ and CdSe (2$4 \mathrm{~nm}$ ) nanoparticles also show that their growth begins with a highly disordered shell and a crystalline core. ${ }^{16,17}$ TEM studies of the early stages of nanocrystallization of minerals (e.g., calcium carbonate, calcium phosphate, and iron oxide) show that the nucleation of these materials is initiated by the formation of amorphous NPs that eventually transform into a crystalline NP during growth. ${ }^{18,19}$ Mirsaidov and co-workers presented a three-step nucleation mechanism for NPs from supersaturated aqueous solutions consisting of spinodal decomposition, solidification, and crystallization. ${ }^{20}$ Recently, Tang and co-workers used observations from in situ liquid cell (LC) TEM to propose a mechanism for nanoparticle nucleation and crystallization with three intermediate steps: prenucleation, nucleation, and maturation. ${ }^{21}$ In the prenuclea-

Received: December 17, 2020

Revised: March 25, 2021

Published: April 5, 2021 

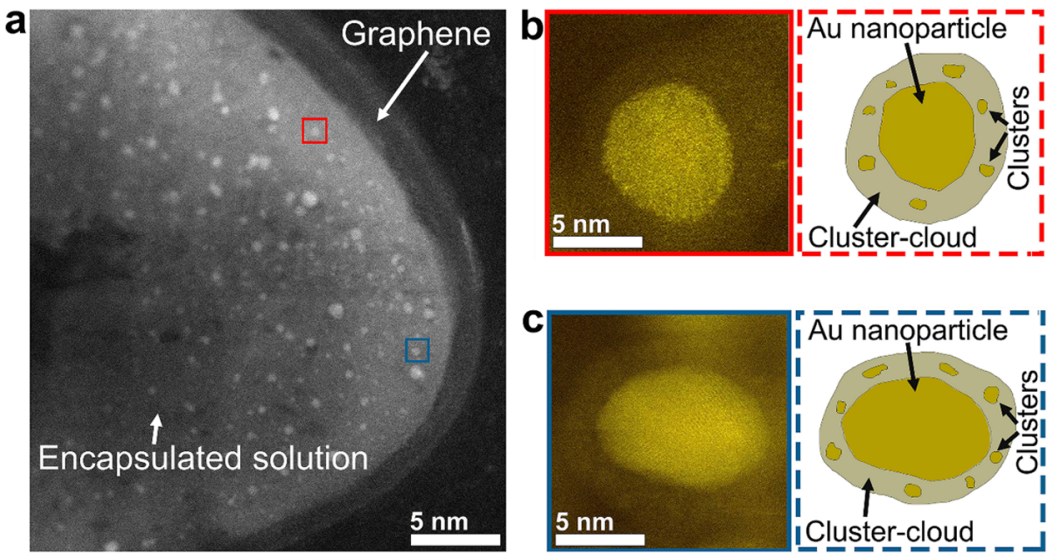

Figure 1. Encapsulated liquid solution between two graphene membranes and the formed Au nanoparticles. (a) Low-magnification ADF-STEM image of the edge of a typical graphene liquid pocket after growth of nanoparticles showing NPs with cluster-clouds. (b,c) High-resolution ADFSTEM images of two selected nanoparticles (marked by red and blue squares in panel a) showing clearly the gold NPs surrounded by a shell consisting of a diffuse cluster-cloud, the schematic representation on the right-hand side of each particle's image, highlighted by dashed squares, illustrates the presence of ultrasmall nanoclusters in the cluster-clouds which appear as density fluctuations. ADF-STEM images of individual NPs are shown in false color.

tion step, a cluster-cloud forms from a supersaturated solution, which in the nucleation step condenses to establish a poorly crystallized nanoparticle. This precursor phase then undergoes multiple out-and-in relaxations involving the exchange of matter with the cluster-cloud, to finally form a well-crystallized NP in the maturation step. ${ }^{21}$ These studies suggest intermediate steps of crystallization; however, until now the underlying detailed, atomic mechanisms of nanocrystallization remain largely unknown. For example, the study of clustercloud induced Pd nanocrystallization made by Jin et al. does not reveal the exact atomic mechanism of matter exchange between the crystal and cluster-cloud but rather speculates that the clusters separation and aggregation could be responsible for the observed out-and-in relaxation. ${ }^{21}$ Here, we present a real-time observation at the atomic level reveals the exact pathways of matter exchange during the out-and-in relaxation processes.

Liquid cell scanning/transmission electron microscopy (LC$\mathrm{S} / \mathrm{TEM}$ ) is an emerging technology allowing direct and realtime observations of dynamic processes in solution. ${ }^{22-27}$ Conventional LCs commonly consist of two $50 \mathrm{~nm}$ thick amorphous silicon nitride membranes, which confine the liquid sample in a volume determined by spacers, ranging from 50 $\mathrm{nm}$ to $5 \mu \mathrm{m}$. So far the method has successfully been applied to observe chemical reactions and the behavior of nanomaterials in solution at the nanometer scale, including nucleation, ${ }^{28-33}$ growth, ${ }^{34-47}$ self-assembly, ${ }^{48-52}$ and particle degradation. ${ }^{53-55}$ When the cell is inside the TEM, the pressure difference between the interior of the liquid cell and the microscope vacuum causes the $\sim 50 \mathrm{~nm}$ thick silicon nitride membranes to bulge out, resulting in a liquid thickness greater than the designed spacing between the two chips. Since the thickness of the LC determines the resolution, the bulging of the membranes further decreases the image resolution. ${ }^{56,57}$ Alternatively, noncommercial graphene liquid cells (GLCs) provide the opportunity to observe nanoparticles at the true atomic level. Graphene sheets are composed of a few monolayers with an overall thickness below $1 \mathrm{~nm}$, allowing the imaging of liquid samples at the Ångström level. The impermeability and flexibility of graphene enables the entrapment of liquid without risk of leakage to the surrounding vacuum environment of the electron microscope. Moreover, the chemical neutrality and electrical conductivity of graphene allows the study of chemical reactions inside GLCs while mitigating radiolysis and charge effects. ${ }^{58,59}$ Here, we use optimized GLCs (Supporting Information (SI) Figure S1) for in situ liquid-phase S/TEM to investigate the early stages of gold NP formation from supersaturated aqueous solution via in situ high-resolution STEM imaging. We study the crystallization mechanism and unravel the atomic details of the outand-in relaxation mechanisms occurring in the crystal, the cluster-cloud, and at the interface between them.

In the work described in this Letter, an aqueous solution of 5 $\mathrm{mM} \mathrm{NaAuCl}{ }_{4}$ is encapsulated between two graphene layers to study the nucleation and growth of $\mathrm{Au}$ nanoparticles. The high-energy electron irradiation in TEM affects the solvent solution by generating radical species. The radiolysis of water from the high-energy electron beam results in the creation of well-established primary products: hydrated electrons $\left(\mathrm{e}_{\mathrm{h}}{ }^{-}\right)$, hydrogen radicals $\left(\mathrm{H}^{\bullet}\right)$, hydroxyl radicals $\left(\mathrm{HO}^{\bullet}\right)$, hydrogen peroxide $\left(\mathrm{H}_{2} \mathrm{O}_{2}\right)$, hydronium ions $\left(\mathrm{H}_{3} \mathrm{O}^{+}\right)$, hydroperoxyl radicals $\left(\mathrm{HO}_{2}{ }^{\circ}\right)$, and hydrogen $\left(\mathrm{H}_{2}\right)$. Strong reducing agents, such as $\mathrm{e}_{\mathrm{h}}{ }^{-}$and $\mathrm{H}^{\bullet}$, react with complex ions of most metals at diffusion controlled rates. Uniformly distributed within the irradiated area, these agents reduce the metal ions to zerovalent metal atoms or atom clusters. ${ }^{60,61}$ The reduction of the encapsulated Au precursor by the electron beam thus triggers nucleation and growth of Au nanoparticles as shown in Figure S2 and Movie S1. The time $t=0 \mathrm{~s}$ in the video represents the starting point of imaging in that area after having optimized the experimental conditions in a different area to minimize the beam effect in the area of observation. The electron dose and the imaging conditions were optimized to enable the growth of gold nanoparticles with kinetics suitable to track the nucleation without inducing unwanted beam effects and particle coalescence (electron dose rate $=298$ electrons $/ \AA^{2}$ s and recording rate $=2$ frames $/ \mathrm{s}$ ). Figure $1 \mathrm{a}$ is a low-magnification annular dark-field (ADF) STEM image of the area of interest, which was chosen at the edge of a liquid pocket where the liquid layer is at its thinnest $(<50 \mathrm{~nm})$ in order to achieve atomic resolution. 

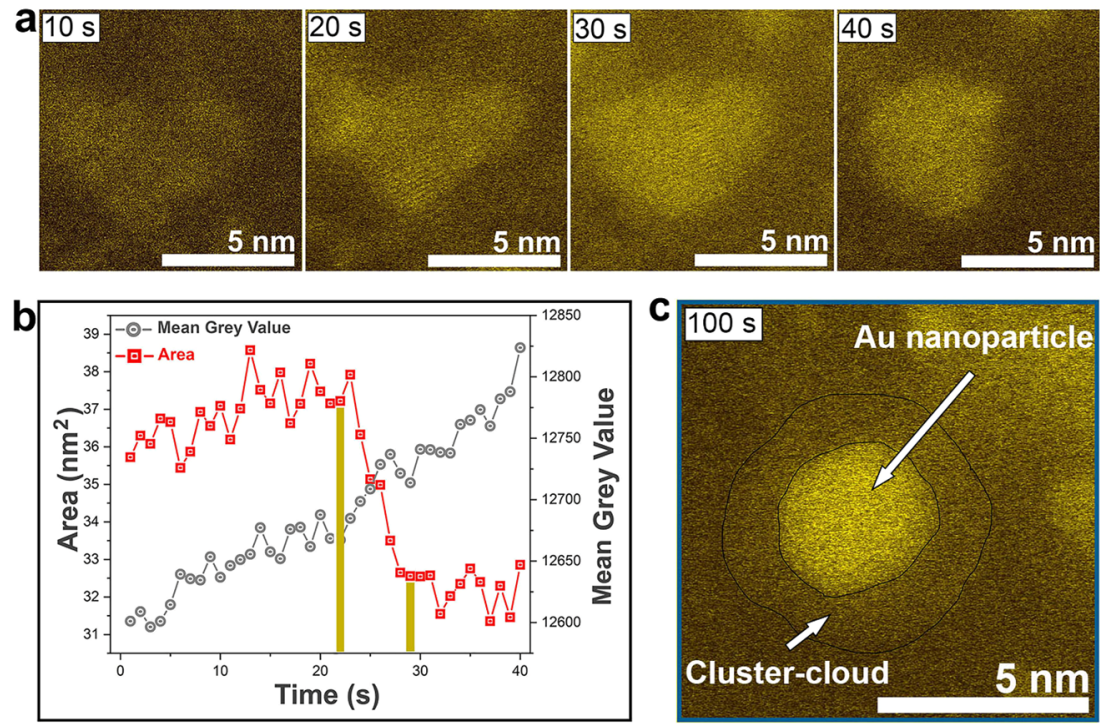

Figure 2. Gold nanocrystal prenucleation and nucleation processes via cluster-cloud condensation. (a) Sequential ADF-STEM images, showing the real time transformation of a cluster-cloud to a gold nanoparticle. (b) Projected Au rich area and mean gray value as a function of time during the condensation process. The yellow lines indicate the time interval, within which the Au rich area suddenly shrinks. Images are shown in false color. (c) ADF-STEM images of the particle formed after condensation of the initial cluster-cloud.

The time sequence of ADF-STEM images in Figure 2a shows the prenucleation and nucleation of a selected $\mathrm{Au}$ nanoparticle, using a false color representation to highlight the $\mathrm{Au}$ rich areas. Here, the region with a higher contrast formed at $t=10 \mathrm{~s}$ corresponds to an increase in concentration of reduced $\mathrm{Au}$ atoms from the initially homogeneous solution. After $20 \mathrm{~s}$, the initial nucleation occurs by the aggregation of the clusters to form a condensed cluster-cloud. Then a sudden collapse of the cluster-cloud occurs and generates a distinct particle surrounded by its disordered shell formed by the remains of the original cluster-cloud. These observations are in line with Jin et al.'s reported data of palladium nanoparticle growth. ${ }^{21}$ We tracked the transformation of the projected Au rich area and the mean gray value of the cluster-cloud as a function of time during the condensation period (Figure $2 b$ ). The plot thus visualizes quantitatively, the point in time when the $\mathrm{Au}$ rich area suddenly shrinks, which is accompanied by a continual increase of its mean gray value (region between two yellow lines in Figure 2b). Shrinkage and densification clearly indicate condensation of the cluster-cloud. A video with more details about the real time prenucleation and nucleation processes can be found in Movie S2.

A typical high-resolution ADF-STEM image of the formed $\mathrm{Au}$ particle after $100 \mathrm{~s}$, presented in Figure $2 \mathrm{c}$, shows that the nanoparticle is surrounded by a diffuse shell, that acts as an active cluster-cloud in the subsequent maturation process. In fact, the particle surrounded by the shell appears to be typical for the investigated system which is supported by additional ADF-STEM observations of other nanocrystals that confirm this condensation process. Indeed, Figure $1 b, c$ shows highresolution ADF-STEM images of two representative $\mathrm{Au}$ nanoparticles (marked by red and blue squares in Figure 1a respectively). Based on the favorable image contrast for heavy elements in ADF-STEM, the analysis of the cluster-cloud shows ultrasmall clusters below $1 \mathrm{~nm}$ in size, which surround particles of varying sizes, as schematically illustrated in Figure 1b,c. Because of the reduced Au density, the contrast of the cluster-cloud is lower compared to the contrast of the $\mathrm{Au}$ nanocrystal. In addition, every cluster-cloud consists of a diffuse shell containing small dots with contrasts similar to the contrast intensities of the $\mathrm{Au}$ nanoparticles. These localized areas of high image intensity are Au nanoclusters and/or single $\mathrm{Au}$ atoms.

Continuous transfer of matter between the cluster-cloud and the $\mathrm{Au}$ nanoparticles is documented in Figure 3 (Movie S3, Supporting Information). The real time observation using ADF-STEM reveals two main ripening processes. First, the $\mathrm{Au}$ nanoparticle releases matter to form the cluster-cloud (Figure $3 a)$. Then, in a second step the released matter is readsorbed from the cluster-cloud by the core-nanoparticle (Figure 3c). Using high-resolution ADF-STEM imaging combined with fast Fourier transforms (FFTs) (Figure S3; Movie S3), we examined in real-time the changes of the particle's crystallinity during this process. Figure 3a shows snapshots from Movie S3. The first ADF-STEM image of this series shows a poorly crystalline (polycrystalline) nanocrystal (inset FFT: top left Figure 3a) that starts losing matter. The released matter does not dissolve in the solution but instead contributes to the cloud surrounding the core nanoparticle $(t=135-165 \mathrm{~s})$. The matter expelled as small clusters $(\sim 1 \mathrm{~nm}$ in size $)$ is indicated by white arrows in Figure 3a. These expelled nanoclusters slowly dissolve in the cluster-cloud, which continues growing (Figure S3a), while at the same time the core of the particle shrinks. This process is named out-process. ${ }^{21}$ The cluster-cloud appears to be amorphous (FFT insets in Figure S3b, Supporting Information). Figure $3 \mathrm{c}$ shows that after the outprocess, the matter dissolved in the cluster-cloud starts gradually migrating toward the core-nanocrystal. This process of cluster adsorption is named in-process. ${ }^{21}$ In order to show that the formation of the cluster-cloud is not limited to thin liquid layers, we have studied particles at both the center and the edge of a liquid pocket. We do not observe any differences in the formation of the cluster-cloud, shown in Figure S4b,c, indicating that the availability of bulk solution around the particle does not influence the crystallization process. These results are in line with findings of cluster-cloud mediated 

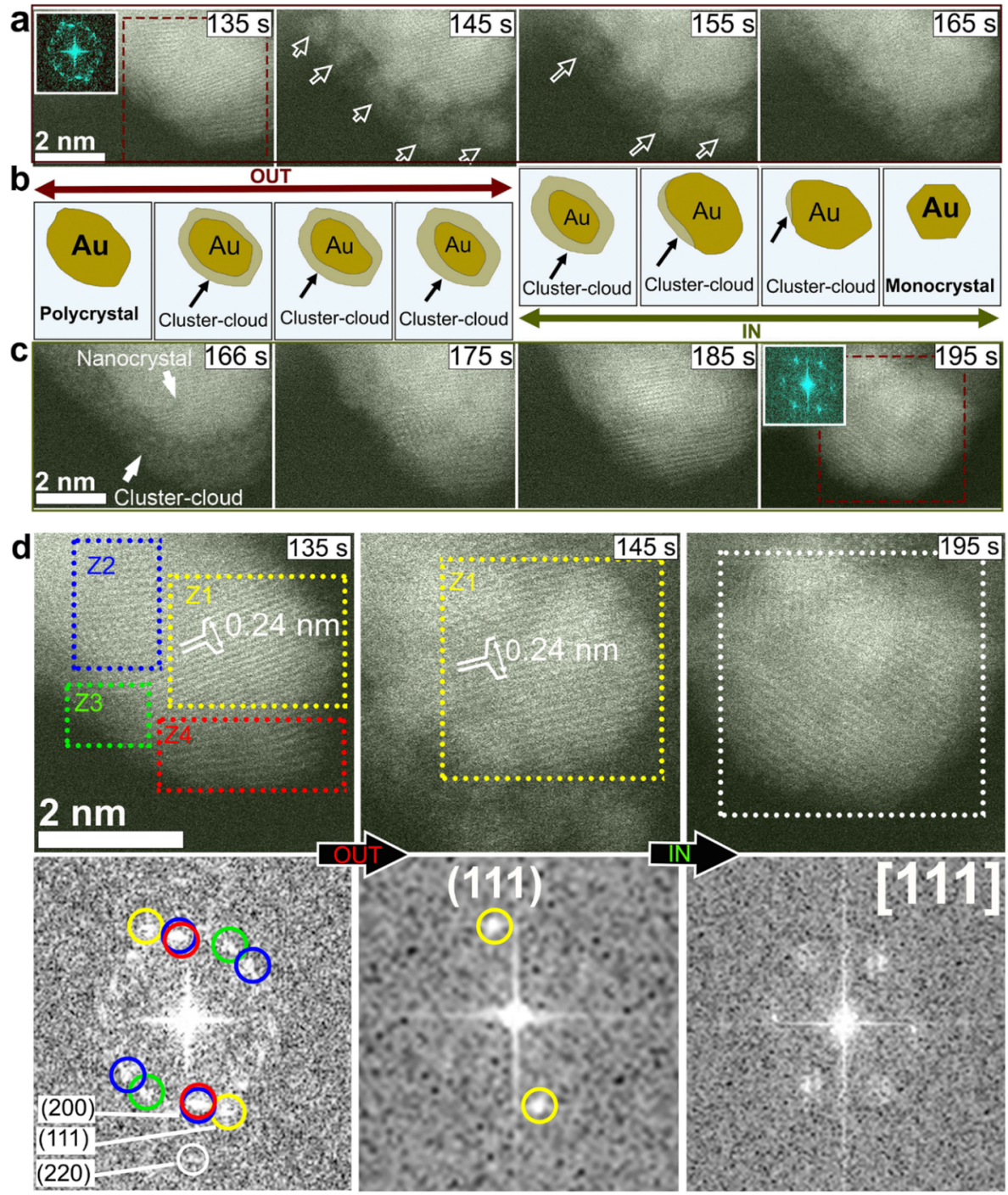

Figure 3. Gold nanocrystal relaxation and growth processes via cluster-cloud. (a) Sequential ADF-STEM images showing the typical out-process with an inset FFT pattern (top left) of the cropped square regions (red), showing the polycrystallinity of the initial nanocrystal seed. (b) Sequential schematic representation series showing the complete cluster-cloud mechanism of out- and in-processes. (c) Sequential ADF-STEM images showing the typical in-process with an inset FFT pattern (top right of the sequences) of the cropped square region (red), revealing the monocrystallinity of the crystal after several out-and-in relaxation steps. The snapshots in panels a and c are extracted from Movie S3. (d) Highresolution ADF-STEM images (top) and their corresponding FFT patterns (bottom) of the nanocrystal obtained after nucleation and after outand-in processes documenting the maturation phase of the nanoparticle based on the exchange of matter with the cluster-cloud. ADF-STEM images are shown in false color.

crystallization of Pd nanoparticle in a silicon nitride-based liquid cell, where the liquid thickness is in excess of $100 \mathrm{~nm} .^{21}$

The sequences of ADF-STEM images in Figure 3c extracted from Movie S3 shows that the core nanoparticle expands while the size of the cloud decreases (more detail in Figure S3b). This clearly shows that the matter expelled from the polycrystalline nanocrystal to the cluster-cloud returned to participate in the particle growth, which gradually becomes a distinct monocrystalline particle (FFT in the inset top right Figure 3c). Both series in Figure 3a,c shows that at $t=135 \mathrm{~s}$, the $\mathrm{Au}$ nanoparticle starts as a particle consisting of different lattice orientations, indicating a polycrystalline nature during the out-process, while in the following growth step the crystallinity of the particle gradually increases to form a monocrystalline particle. The monocrystallinity is preserved during the in-process, where the particle at $t=195 \mathrm{~s}$ shows a high degree of crystallinity (FFT in inset). Figure $3 \mathrm{~b}$ shows a schematic representation summarizing this typical process of out-and-in relaxation pathways allowing the maturation of the nanoparticle from a polycrystalline nanocrystal to a single, welldefined nanocrystal. The question that arises is, how the matter in the cluster-cloud can be adsorbed by the core nanocrystal to improve its crystallinity in this ripening process.

Figure $3 \mathrm{~d}$ shows two typical high-resolution ADF-STEM images and their corresponding FFT patterns of the particle before and after multiple out- and in-processes. The highresolution ADF-STEM image at $t=135 \mathrm{~s}$ corresponds to the primary nanocrystal produced by nucleation via condensation, which exhibited a polycrystalline structure. This is revealed by the corresponding FFT pattern, where the coexistence of more than four different lattice orientations inside the particle is documented. At $t=145 \mathrm{~s}$ and during the out-process, the particle shrinks in size while its crystallinity improves. The ADF-STEM image and its corresponding FFT pattern of the 


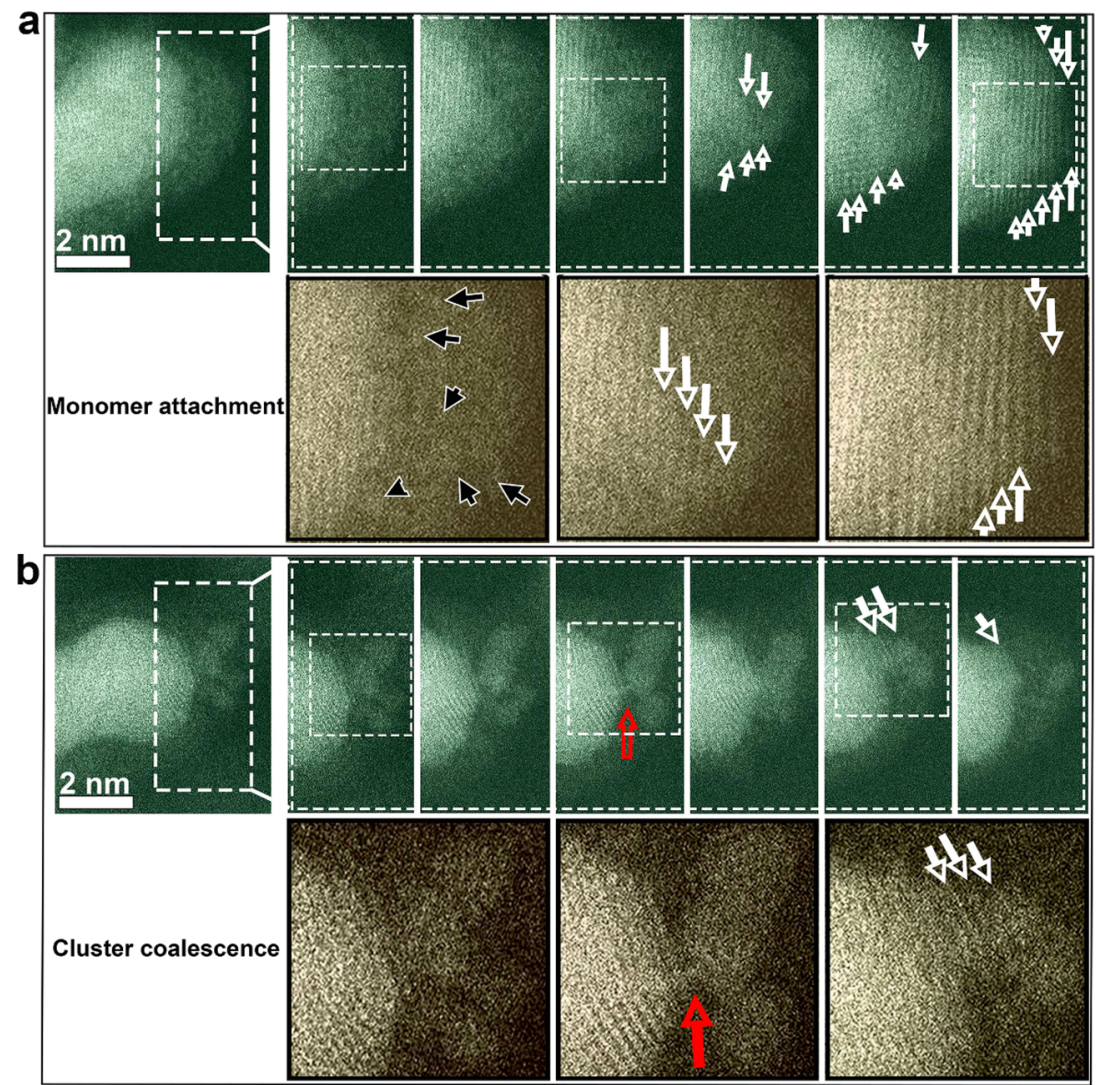

Figure 4. In-relaxation process of a poorly crystallized particle to form a single crystal. (a) Sequential high-resolution ADF-STEM images of the interface between core-particle and the cluster-cloud, showing single atoms and ultrasmall clusters (black arrows in the zoom-in) dissolved in the cluster-cloud attaching to the core-particle to form new atomic layers (white arrows). (b) Sequential high-resolution ADF-STEM images of the interface between core-particle and the cluster-cloud, showing clusters, which first form in the cluster-cloud, and then attach to the core-particle by bridge-induced (red arrow) coalescence to form new epitaxial atomic layers (white arrows). Images are shown in false color.

particle after $145 \mathrm{~s}$ shows only one domain of the crystal lattice. This improvement in crystallinity means that disordered and amorphous areas are expelled during the outprocess, while maintaining the dominant crystallized central part of the primary particles (Zone $\mathrm{Z} 1$ ). In the next step, the matter expelled into the cluster-cloud is back-adsorbed to the nanocrystal, which implies that the particle grows and it becomes monocrystalline at $t=195 \mathrm{~s}$. The corresponding FFT pattern at $195 \mathrm{~s}$ shows a monocrystalline pattern oriented approximately along [111] zone-axis direction.

To consolidate our findings and account for electron beam effects, we repeated the experiment at two lower electron dose rates of 170 and 90 electrons/ $\AA^{2}$ s, Figure S5. We observe the same out-and-in relaxation process at lower electron dose rates as we did at the high electron dose rate (19072 electrons $/ \AA^{2}$ s), indicating the process is independent of the electron beam, and we do not expect that an electron beam-induced temperature increase should be of significance. ${ }^{20}$ The out-and-in relaxation process also occurred while the electron beam was blanked, discounting this phenomenon as beam induced (see Figure S5 in Supporting Information for more details). We have documented multiple occurrences of the out-and-in relaxation process in Figure S4, where cluster-clouds exist for particles with different sizes and shapes. Furthermore, this nonclassical growth process is observed at varying $\mathrm{NaAuCl}_{4}$ concentrations $(2.5,5,20 \mathrm{mM})$ as shown in Figure S6. This confirms that nanoparticle crystallization via the cluster-cloud is independent of electron beam irradiation and is a universal phenomenon in the investigated system.

Our study also allows for deriving information about the composition of the cluster-cloud. High-resolution ADF-STEM imaging reveals the coexistence of two types of clusters in the cluster-cloud; clusters of around $1 \mathrm{~nm}$ in size and ultrasmall diffuse clusters $<1 \mathrm{~nm}$ (Figure S3, Supporting Information). The real time observation (Movie S3) shows that both categories of clusters were ejected simultaneously into the cluster-cloud. Then, the larger, unstable clusters can be dissolved in the cluster-cloud as single atoms and/or ultrasmall clusters.

We followed in real-time at atomic level the evolution of the interface between the cluster-cloud and the core nanoparticle during the out-and-in relaxation processes. Regarding the growth of the nanocrystal being fed by the Au atoms in the cluster-cloud, we can distinguish two processes. (i) Inrelaxation by means of monomer attachment: single atoms and ultrasmall clusters, dispersed in the cluster-cloud during the out-process, are observed to reattach to the seed crystal during the in-process (Movie S3). Figure 4a shows a series of high-resolution ADF-STEM images acquired during the inprocesses of the nanocrystal in Movie S3, the zoom-in on the interface between the nanocrystal and the cluster-cloud shows amorphous $\mathrm{Au}$ and/or ultrasmall clusters attached to the nanocrystal, leading to the formation of new atomic layers, highlighted with white arrows. The monomer attachment 


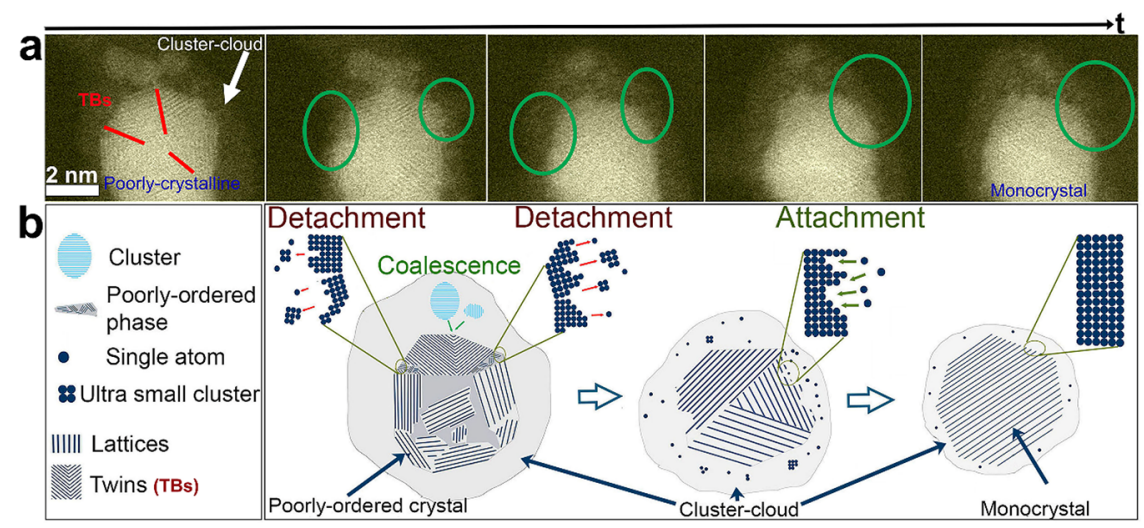

Figure 5. Multiple relaxation mechanisms occurring simultaneously. (a) Sequential high-resolution ADF-STEM images (shown in false color) of a core nanoparticle surrounded by a cluster-cloud, documenting the coalescence of the crystal with distinct clusters, which occurs simultaneously with monomer detachment and attachment (green circles). (b) Schematic representation showing step-by-step the nanocrystallization of the particle via multiple simultaneously active processes: coalescence, monomer detachment, and monomer attachment. In the diagrams, the arrows indicate the direction of the monomers ( red $=$ detachment and green $=$ attachment). The blue lines indicate the lattice planes, the gray part inside the particle indicates the amorphous part, and the gray outside indicates the cluster-cloud.

governing this in-process allows the formation of a monocrystalline particle.

The other growth process involves larger clusters: (ii) Inrelaxation by means of rapid coalescence proces. In this second type of in-process, rather stable clusters are formed via the aggregation of atoms and ultrasmall clusters inside the clustercloud (Figure S7). A possible explanation for the stability of these clusters can be found in the magic cluster size of classical crystallization theory. The extraordinary stability of these magic clusters is given by the geometric closed-shell structure, where the cluster forms a close-packed structure of atoms with no vacancies at the surface of a symmetric polyhedron along with a favorable electronic structure. Thus, for a certain number of atoms the shape and structure of the cluster reaches a high stability. These structures have been observed in clusters of gold and other platinum group metals. ${ }^{62}$ In our study, nanocrystals are observed to expel matter in the cluster-cloud, where two types of clusters can be distinguished; one type continues to exist and grows in the cluster-cloud and then aggregates by rapid coalescence. The second type is unstable and dissolves in the cluster-cloud. This instability relates to an unfavorable number of atoms, that is, the unstable shape and structure trigger dissolution of the cluster into the clustercloud, and the dissolved atoms eventually adsorb to the crystal via monomer attachment. Figure S3 shows the dissolution of unstable nanoclusters and Figure S8 shows the stable expelled nanoclusters.

The real-time observation of one of these larger clusters (Movie S4) shows a typical in-process based on rapid coalescence. Figure S8 is a series of high-resolution ADFSTEM images showing step-by-step the coalescence of four nanoclusters (in the cluster-cloud) with the core-nanocrystal. At the beginning of the observation $(t=100 \mathrm{~s})$, the clustercloud shows no clusters, then at $t=104 \mathrm{~s}$ four nanoclusters have formed inside the cluster-cloud. These clusters coalesce with the core-nanocrystal, which leads to a rapid growth step of this central nanocrystal. Figure $4 \mathrm{~b}$ shows the zoom-in at the interface between the cluster-cloud and the core-crystal showing step-by-step the coalescence of four nanoclusters with the core-nanocrystal. At the beginning of coalescence, the contact between clusters and the core-crystal is via nanobridgeinduced contact (red arrow in Figure 4b). Remarkably, the nanoclusters' lattice orientation aligns after contact with the core-crystal (zoom-in Figure 4b). Afterword, the connected nanoclusters coalesce totally to the core-crystal, resulting in new crystalline phase that is added to the core-nanocrystal (white arrows in Figure 4b). We examined in real-time the changes of the particle's crystallinity during this process using high-resolution ADF-STEM imaging combined with FFTs (Figure S8, bottom). During the coalescence, the crystallinity of the nanocrystal improves, and there was no evidence of lattice distortion.

Compared to in-relaxation governed by monomer attachment-induced crystallization and shape rearrangement, which leads directly to a well-defined crystalline nanoparticle as described above, the in-relaxation by means of coalescence showed an incomplete crystallinity improvement, which needs to be completed with monomer attachment to bring the crystal from a poorly crystalline to a monocrystalline state. Our observations (Figure 5) show that this process occurs simultaneously with an out-process, where the poorly ordered and amorphous phases are expelled to the cluster-cloud, while the nanoclusters attach to the core-crystal. Figure 5a shows a series of ADF-STEM images of one particle undergoing coalescence-induced crystallization. During the coalescence process, detachment of monomers occurs simultaneously, allowing for an intrinsic relaxation (e.g., strain relaxation induced by twin boundary migration (red solid lines in Figure 5a)). This leads to a higher degree of crystallinity and shape reconstruction as well as elimination of defects (Figure 5a, right). The starting nanoparticle contains different lattice orientations and disordered and amorphous parts (Figure 5a, left). During the coalescence of the clusters with the nanoparticle, the shape and the structure of the particle fluctuate from one state to another, and the crystal simultaneously ejects the disordered and the amorphous phases via monomer detachment (out-process), eventually leading to full relaxation of the crystal (Movie S4). Figure 5b shows a diagram detailing the coalescence and monomer detachment; the first diagram depicts a disordered nanoparticle, which is simultaneously involved in a coalescence process with nanoclusters and the detachment of monomers from the disordered phases. This leads to a better-ordered nanocrystal without defects and without amorphous phase (the 
middle diagram). After complete ejection of the disordered phase, the monomers forming the cluster-cloud attach to the cluster to form a final monocrystalline particle (right diagram).

In summary, our work on in situ STEM using GLCs offers direct atomic level and real time observation of the very early stages of $\mathrm{Au}$ nanoparticle formation in a supersaturated aqueous solution. Our observations clearly show that during nanoparticle growth and the so-called maturation phase of the nanocrystal via multiple out-and-in relaxation processes, the exchange of matter between the nanocrystal and its clustercloud involves different pathways leading to a mature monocrystalline nanoparticle. The observed out-process is based on expelling matter from the poorly crystalline parts of the initial seed nanoparticle into the diffuse cluster-cloud. These expelled clusters dissolve in the cluster-cloud and eventually readsorb on the crystal in two distinct pathways: (i) in-process via monomer attachment and (ii) in-process via cluster coalescence. We showed that the in-process by means of monomer attachment is very efficient and sufficient to rapidly bring the crystal from a poorly crystalline phase to a monocrystalline phase. However, cluster coalescence is not sufficient for proper maturation of the crystal, as it is only important for recrystallization and shape transformation, whereas the cluster coalescence does not lead to a complete monocrystalline nanoparticle. The other, simultaneously active process, namely, triggered by monomer attachment, eventually allows the formation of the complete monocrystalline phase.

Although a previous study conducted by Jin et al. gave an overview of the role of cluster-cloud in nanocrystallization of Pd naocrystals, ${ }^{21}$ here we present the first insight at the atomic level of the out-and-in relaxation process and document the underlying atomic mechanisms. Our results reveal the universality of cluster-cloud induced crystallization processes and provide an exact atomistic description of the mechanisms governing nanocrystallization via cluster-cloud formation. By imaging in situ, real-time observations reveal the complexity of the out-and-in relaxation process, especially at the interface of the nanoparticle and cluster-cloud, where different relaxation processes can lead to nanoparticle maturation.

The findings reported in this Letter confirm the previously suggested growth of particle via cluster-clouds and expand the current understanding by uncovering the detailed, atomic scale mechanisms of nonclassical nucleation and crystallization theory. The atomic-resolution insights revealed in our study are important to understand nanoparticle growth mechanisms and the nanocrystallization pathway of nanocrystals in the maturation phase and thus provide an in-depth insight into nanomaterial's synthesis for the particular system investigated.

\section{ASSOCIATED CONTENT}

\section{s) Supporting Information}

The Supporting Information is available free of charge at https://pubs.acs.org/doi/10.1021/acs.nanolett.0c04965.

Details for the sample preparation, GLCs fabrication and methods for analyses, Figures S1-S5 (PDF)

In-situ STEM movie showing the growth of $\mathrm{Au}$ nanoparticles (AVI)

In-situ STEM movie showing the condensation processes to form a Au nanocrystal (AVI)

In-situ STEM movie showing the relaxation processes of a Au nanocrystal (AVI)
In-situ STEM movie showing the in-processes via coalescence of clusters (AVI)

\section{AUTHOR INFORMATION}

\section{Corresponding Authors}

Walid Dachraoui - Electron Microscopy Center, Empa-Swiss Federal Laboratories for Materials Science and Technology, CH-8600 Dübendorf, Switzerland; (1) orcid.org/0000-00017599-5856; Email: walid.dachaoui@empa.ch

Rolf Erni - Electron Microscopy Center, Empa-Swiss Federal Laboratories for Materials Science and Technology, CH-8600 Dübendorf, Switzerland; ㅇ orcid.org/0000-0003-2391-

5943; Email: rolf.erni@empa.ch

\section{Authors}

Debora Keller - Electron Microscopy Center, Empa-Swiss Federal Laboratories for Materials Science and Technology, CH-8600 Dübendorf, Switzerland; (1) orcid.org/00000003-2343-7275

Trond R. Henninen - Electron Microscopy Center, EmpaSwiss Federal Laboratories for Materials Science and Technology, CH-8600 Dübendorf, Switzerland; (1) orcid.org/0000-0002-1271-9632

Olivia J. Ashton - Electron Microscopy Center, Empa-Swiss Federal Laboratories for Materials Science and Technology, CH-8600 Dübendorf, Switzerland; 이이.org/00000002-0886-2110

Complete contact information is available at:

https://pubs.acs.org/10.1021/acs.nanolett.0c04965

\section{Author Contributions}

W.D. and R.E. conceived the idea and wrote the manuscript. W.D. fabricated the liquid cells, performed the liquid-cell experiments and realized the in situ STEM characterizations. W.D., D.K., T.R.H., and O.J.A. carried out the data analysis. R.E. supervised the project. All authors discussed the results and commented on the manuscript.

Notes

The authors declare no competing financial interest.

\section{ACKNOWLEDGMENTS}

This work received funding from European Research Council (ERC) under the EU's Horizon 2020 research and innovation program under Grant 681312.

\section{REFERENCES}

(1) Zhao, R. B.; Liu, X. Y.; Yang, X. Y.; Jin, B.; Shao, C. Y.; Kang, W. J.; Tang, R. K. Metal-Organic Frameworks for Separation. Adv. Mater. 2018, 30, 1801304.

(2) Gwo, S.; Chen, H. Y.; Lin, M. H.; Sun, L. Y.; Li, X. Q. Nanomanipulation and controlled self-assembly of metal nanoparticles and nanocrystals for plasmonics. Chem. Soc. Rev. 2016, 45, $5672-5716$.

(3) Fan, Z. X.; Zhang, H. Template Synthesis of Noble Metal Nanocrystals with Unusual Crystal Structures and Their Catalytic Applications. Acc. Chem. Res. 2016, 49, 2841-2850.

(4) Jiang, C.; Hosono, E.; Zhou, H. Nanomaterials for lithium ion batteries. Nano Today 2006, 1, 28-33.

(5) Zhang, P. N.; Xi, C. X.; Feng, C. H.; Xia, B.; Wang, D. Y.; Tao, $\mathrm{X}$. T. Transition metal ion-assisted synthesis of monodisperse, quasispherical gold nanocrystals via citrate reduction. CrystEngComm 2014, $16,5268-5274$.

(6) Jeevanandam, J.; Barhoum, A.; Chan, Y. S.; Dufresne, A.; Danquah, K. M. Review on nanoparticles and nanostructured 
materials: history, sources, toxicity and regulations. Beilstein J. Nanotechnol. 2018, 9, 1050-1074.

(7) Thanh, T. K. N.; Maclean, N.; Mahiddine, S. Mechanisms of Nucleation and Growth of Nanoparticles in Solution. Chem. Rev. 2014, 114, 7610-7630.

(8) Khan, I.; Saeed, K.; Khan, I. Nanoparticles: Properties, applications and toxicities. Arabian J. Chem. 2019, 12, 908-931.

(9) Völkle, C. M.; Gebauer, D.; Cölfen, H. High-resolution insights into the early stages of silver nucleation and growth. Faraday Discuss. 2015, 179, 59-77.

(10) Kellermeier, M.; Gebauer, D.; Melero-Garcia, E.; Drechsler, M.; Talmon, Y.; Kienle, L.; Colfen, H.; Garcia-Ruiz, J. M.; Kunz, W. Colloidal Stabilization of Calcium Carbonate Prenucleation Clusters with Silica. Adv. Funct. Mater. 2012, 22, 4301-4311.

(11) Chattopadhyay, S.; Erdemir, D.; Evans, J. M. B.; Ilavsky, J.; Amenitsch, H.; Segre, C. U.; Myerson, A. S. SAXS Study of the Nucleation of Glycine Crystals from a Supersaturated Solution. Cryst. Growth Des. 2005, 5, 523-527.

(12) Young, N. P.; Li, Z. Y.; Chen, Y.; Palomba, S.; Di Vece, M.; Palmer, R. E. Weighing Supported Nanoparticles: Size-Selected Clusters as Mass Standards in Nanometrolog. Phys. Rev. Lett. 2008, 101, 246103.

(13) Sitja, G.; Le Moal, S.; Marsault, M.; Hamm, G.; Leroy, F.; Henry, R. C. Transition from Molecule to Solid State: Reactivity of Supported Metal Clusters. Nano Lett. 2013, 13, 1977-1982.

(14) Marks, L. D. Experimental studies of small particle structures. Rep. Prog. Phys. 1994, 57, 603-649.

(15) Chen, X.; Samia, A. C. S.; Lou, Y.; Burda, C. Investigation of the crystallization process in $2 \mathrm{~nm}$ CdSe quantum dots. J. Am. Chem. Soc. 2005, 127, 4372-4375.

(16) Gilbert, B.; Huang, F.; Zhang, H.; Waychunas, G. A.; Banfield, J. F. Nanoparticles: strained and stiff. Science 2004, 305, 651-654.

(17) Masadeh, A. S.; Božin, E. S.; Farrow, C. L.; Paglia, G.; Juhas, P.; Billinge, S. J. L.; Karkamkar, A.; Kanatzidis, M. G. Quantitative sizedependent structure and strain determination of CdSe nanoparticles using atomic pair distribution function analysis. Phys. Rev. B: Condens. Matter Mater. Phys. 2007, 76, 115413.

(18) Gebauer, D.; Völkel, A.; Cölfen, H. Stable prenucleation calcium carbonate clusters. Science 2008, 322, 1819-1822.

(19) Pouget, E. M.; Bomans, P. H. H.; Goos, J. A. C. M.; Frederik, P. M.; De With, G.; Sommerdijk, N.A. J. M. The initial stages of template-controlled $\mathrm{CaCO}_{3}$ formation revealed by cryo-TEM. Science 2009, 323, 1455-1458.

(20) Loh, N. D.; Sen, S.; Bosman, M.; Tan, S. F.; Zhong, J.; Nijhuis, C. A.; Král, P.; Matsudaira, P.; Mirsaidov, U. Multistep nucleation of nanocrystals in aqueous solution. Nat. Chem. 2017, 9, 77-82.

(21) Jin, B.; Wang, Y.; Liu, Z.; France-Lanord, A.; Grossman, J. C.; Jin, C.; Tang, R. Revealing the Cluster-cloud and its Role in Nanocrystallization. Adv. Mater. 2019, 31, 1808225.

(22) Alloyeau, D.; Dachraoui, W.; Javed, Y.; Belkahla, H.; Wang, G.; Lecoq, H.; Ammar, S.; Ersen, O.; Wisnet, A.; Gazeau, F.; Ricolleau, C. Unravelling Kinetic and Thermodynamic Effects on the Growth of Gold Nanoplates by Liquid Transmission Electron Microscopy. Nano Lett. 2015, 15, 2574-2581.

(23) Lutz, L.; Dachraoui, W.; Demortière, A.; Johnson, L. R.; Bruce, P. G.; Grimaud, A.; Tarascon, J. M. Operando Monitoring of the Solution-Mediated Discharge and Charge Processes in a $\mathrm{Na}-\mathrm{O}_{2}$ Battery Using Liquid-Electrochemical Transmission Electron Microscopy. Nano Lett. 2018, 18, 1280-1289.

(24) Zhang, Y.; Keller, D.; Rossell, D. M.; Erni, R. Formation of Au Nanoparticles in Liquid Cell Transmission Electron Microscopy: From a Systematic Study to Engineered Nanostructures. Chem. Mater. 2017, 29, 10518-10525.

(25) Keller, D.; Henninen, R. T.; Erni, R. Formation of gold nanoparticles in a free-standing ionic liquid triggered by heat and electron irradiation. Micron 2019, 117, 16-21.

(26) Zheng, H.; Smith, R. K.; Jun, Y. W.; Kisielowski, C.; Dahmen, U.; Alivisatos, A. P. Observation of single colloidal platinum nanocrystal growth trajectories. Science 2009, 324, 1309-1312.
(27) De Jonge, N.; Ross, F. M. Electron microscopy of specimens in liquid. Nat. Nanotechnol. 2011, 6, 695-704.

(28) De Clercq, A.; Dachraoui, W.; Margeat, O.; Pelzer, K.; Henry, C. R.; Giorgio, S. Growth of Pt-Pd Nanoparticles Studied In Situ by HRTEM in a Liquid Cell. J. Phys. Chem. Lett. 2014, 5, 2126-2130.

(29) Song, B.; He, K.; Yuan, Y.; Sharifi-Asl, S.; Cheng, M.; Lu, J.; Saidi, W. A.; Shahbazian-yassar, R. In situ study of nucleation and growth dynamics of Au nanoparticles on $\mathrm{MoS}_{2}$ nanoflakes. Nanoscale 2018, 10, 15809-15818.

(30) Li, J.; Chen, J.; Wang, H.; Chen, N.; Wang, Z.; Guo, L.; Deepak, F. L. In Situ Atomic-Scale Study of Particle-Mediated Nucleation and Growth in Amorphous Bismuth to Nanocrystal Phase Transformation. Adv. Sci. 2018, 5, 1700992.

(31) Wang, M.; Park, C.; Woehl, T. J. Quantifying the Nucleation and Growth Kinetics of Electron Beam Nanochemistry with Liquid Cell Scanning Transmission Electron Microscopy. Chem. Mater. 2018, 30, 7727-7736.

(32) Nielsen, M. H.; Li, D.; Zhang, H.; Aloni, S.; Han, T. Y. J.; Frandsen, C.; Seto, J.; Banfield, J. F.; Cölfen, H.; De Yoreo, J. J. Investigating Processes of Nanocrystal Formation and Transformation via Liquid Cell TEM. Microsc. Microanal. 2014, 20, 425-436.

(33) Nielsen, M. H.; Aloni, S.; De Yoreo, J. J. In situ TEM imaging of $\mathrm{CaCO}_{3}$ nucleation reveals coexistence of direct and indirect pathways. Science 2014, 345, 1158-1162.

(34) Wei, W.; Zhang, H.; Wang, W.; Dong, M.; Nie, M.; Sun, L.; Xu, F. Observing the Growth of $\mathrm{Pb}_{3} \mathrm{O}_{4}$ Nanocrystals by in Situ Liquid Cell Transmission Electron Microscopy. ACS Appl. Mater. Interfaces 2019, 11, 24478-24484.

(35) Ahn, T. Y.; Hong, S. P.; Kim, S. I.; Kim, Y. W. In situ liquid-cell transmission electron microscopy for direct observation of concentration-dependent growth and dissolution of silver nanoparticles. RSC Adv. 2015, 5, 2342-82345.

(36) Cheong, J. Y.; Chang, J. H.; Kim, S. J.; Kim, C.; Seo, H. K.; Shin, J. W.; Yuk, J. M.; Lee, J. Y.; Kim, I. D. In Situ High-Resolution Transmission Electron Microscopy (TEM) Observation of Sn Nanoparticle on $\mathrm{SnO}_{2}$ Nanotubes Under Lithiation. Microsc. Microanal. 2017, 23, 1107-1115.

(37) Evans, J. E.; Jungjohann, K. L.; Browning, N. D.; Arslan, I. Controlled Growth of Nanoparticles from Solution with In Situ Liquid Transmission Electron Microscopy. Nano Lett. 2011, 11, 2809-2813.

(38) Yuk, J. M.; Park, J.; Ercius, P.; Kim, K.; Hellebusch, D. J.; Crommie, M. F.; Lee, J. Y.; Zettl, A.; Alivisatos, A. P. High-Resolution EM of Colloidal Nanocrystal Growth Using Graphene Liquid Cells. Science 2012, 336, 61-64.

(39) Xin, H. L.; Zheng, H. In situ observation of oscillatory growth of bismuth nanoparticles. Nano Lett. 2012, 12, 1470-1474.

(40) Patterson, J. P.; Abellan, P.; Denny, M. S.; Park, C.; Browning, N. D.; Cohen, S. M.; Evans, J. E.; Gianneschi, N. C. Observing the Growth of Metal-Organic Frameworks by in Situ Liquid Cell Transmission Electron Microscopy. J. Am. Chem. Soc. 2015, 137, $7322-7328$.

(41) Chen, Y.-C.; Chen, J.-Y.; Wu, W.-W. In Situ Observation of Au Nanostructure Evolution in Liquid Cell TEM. J. Phys. Chem. C 2017, 121, 26069-26075.

(42) Lin, Y. H.; Chen, J. Y.; Chen, F. C.; Kuo, M. Y.; Hsu, Y. J.; Wu, W. W. In Situ Analysis of Growth Behaviors of $\mathrm{Cu}_{2} \mathrm{O}$ Nanocubes in Liquid Cell Transmission Electron Microscopy. Anal. Chem. 2019, 91, 9665-9672.

(43) Evans, J. E.; Jungjohann, K. L.; Browning, N. D.; Arslan, I. Controlled growth of nanoparticles from solution with in situ liquid transmission electron microscopy. Nano Lett. 2011, 11, 2809-2813.

(44) Qin, F.; Wang, Z.; Wang, Z. L. Anomalous growth and coalescence dynamics of hybrid perovskite nanoparticles observed by liquid-cell transmission electron microscopy. ACS Nano 2016, 10, 9787.

(45) Liang, W.-I; Zhang, X.; Bustillo, K.; Chiu, C.-H.; Wu, W.-W.; $\mathrm{Xu}$, J.; Chu, Y.-H.; Zheng, H. In situ study of spinel ferrite nanocrystal 
growth using liquid cell transmission electron microscopy. Chem. Mater. 2015, 27, 8146-8152.

(46) Parent, L. R.; Robinson, D. B.; Woehl, T. J.; Ristenpart, W. D.; Evans, J. E.; Browning, N. D.; Arslan, I. Direct in situ observation of nanoparticle synthesis in a liquid crystal surfactant template. ACS Nano 2012, 6, 3589-3596.

(47) Zhu, G.; Jiang, Y.; Lin, F.; Zhang, H.; Jin, C.; Yuan, J.; Yang, D.; Zhang, Z. In situ study of the growth of two-dimensional palladium dendritic nanostructures using liquid-cell electron microscopy. Chem. Commun. 2014, 50, 944.

(48) Javon, E.; Gaceur, M.; Dachraoui, W.; Margeat, O.; Ackermann, J.; Saba, M. I.; Delugas, P.; Mattoni, A.; Bals, S.; Van Tendeloo, G. Competing Forces in the Self-Assemblyof Coupled $\mathrm{ZnO}$ Nanopyramids. ACS Nano 2015, 9, 3685-3694.

(49) Sutter, E.; Sutter, P.; Tkachenko, A. V.; Krahne, R.; de Graaf, J.; Arciniegas, M.; Manna, L. In situ microscopy of the self-assembly of branched nanocrystals in solution. Nat. Commun. 2016, 7, 11213.

(50) Touve, M. A.; Carlini, A. S.; Gianneschi, N. C. Self-assembling peptides imaged by correlated liquid cell transmission electron microscopy imaging mass spectrometry. Nat. Commun. 2019, 10, 4837.

(51) Wang, H.; Li, B.; Kim, Y. J.; Kwon, O. H.; Granick, S. Intermediate states of molecular self-assembly from liquid-cell electron microscopy. App Phy Sci. 2019, 117, 1283-1292.

(52) Cepeda-Perez, E.; Doblas, D.; Kraus, T.; de Jonge, N. Electron microscopy of nanoparticle superlattice formation at a solid-liquid interface in nonpolar liquids. Sci. Adv. 2020, 6, 1404.

(53) Elgrabli, D.; Dachraoui, W.; Ménard-Moyon, C.; Liu, X. J.; Begin, D.; Bégin-Colin, S.; Bianco, A.; Gazeau, F.; Alloyeau, D. Carbon Nanotube Degradationin Macrophages: Live Nanoscale Monitoring and Understanding of Biological Pathway. ACS Nano 2015, 9, 10113-10124.

(54) Zhu, G. Z.; Prabhudev, S.; Yang, J.; Gabardo, C. M.; Botton, G. A.; Soleymani, L. In Situ Liquid Cell TEM Study of Morphological Evolution and Degradation of Pt-Fe Nanocatalysts During Potential Cycling. J. Phys. Chem. C 2014, 118, 22111-22119.

(55) Beermann, V.; Holtz, M. E.; Padgett, E.; De Araujo, F. J.; Muller, D. A.; Strasser, P. Real-time imaging of activation and degradation of carbon supported octahedral $\mathrm{Pt}-\mathrm{Ni}$ alloy fuel cell catalysts at the nanoscale using in situ electrochemical liquid cell STEM. Energy Environ. Sci. 2019, 12, 2476-2485.

(56) Yang, J.; Paul, O. Fracture properties of LPCVD silicon nitride thin films from the load-detection of long membranes. Sens. Actuators, A 2002, 97, 520-526.

(57) Ross, F. M.; De Jonge, N. Electron microscopy of specimens in liquid. Nat. Nanotechnol. 2011, 6, 695-704.

(58) Hauwiller, M. R.; Ondry, J. C.; Alivisatos, A. P. Using Graphene Liquid Cell Transmission Electron Microscopy to Study in Situ Nanocrystal Etching. J. Visualized Exp. 2018, 135, 2-9.

(59) Ghodsi, S. M.; Megaridis, C. M.; Shahbazian-Yassar, R.; Shokuhfar, T. Advances in Graphene-Based Liquid Cell Electron Microscopy: Working Principles, Opportunities, and Challenges. Small Methods 2019, 3, 1-16.

(60) Schneider, N. M.; Norton, M. M.; Mendel, B. J.; Grogan, J. M.; Ross, F. M.; Bau, H. H. Electron-Water Interactions and Implications for Liquid Cell Electron Microscopy. J. Phys. Chem. C 2014, 118, 22373-22382.

(61) Schwarz, H. A. Free Radicals Generated by Radiolysis of Aqueous Solutions. J. Chem. Educ. 1981, 58, 101-105.

(62) Lee, J.; Yang, J.; Kwon, S. G.; Hyeon, T. Non-classical nucleation and growth of inorganic nanoparticles. Nature Reviews Materials 2016, 1, 16034. 